\title{
Development of a space-based nulling interferometer to detect and characterize exoplanets
}

\author{
L. Schifano ${ }^{\mathrm{a}}$, D. Defrère ${ }^{\mathrm{b}}$, O. Absil ${ }^{\mathrm{b}}$, J. Loicqa $^{\mathrm{a}}$.
}

${ }^{a}$ Centre Spatial de Liege, STAR institute, University of Liege, Belgium

bPSILab, Astrophysics and Geophysics Institute, STAR institute, University of Liege Belgium
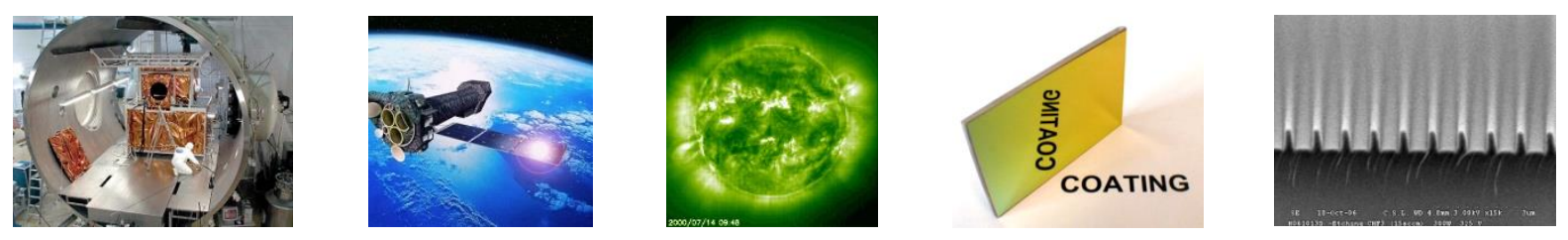


\section{Outline}

- Exoplanetary observation context

- Nulling Interferometry principle

- Target science with small plateform

- Alpha Centauri system

- Proxima b

- Kepler Statistics

- 3 wavelength rages studied (Vis/MWIR/LWIR)

- Results / Mission concept

- Conclusions 


\section{1}

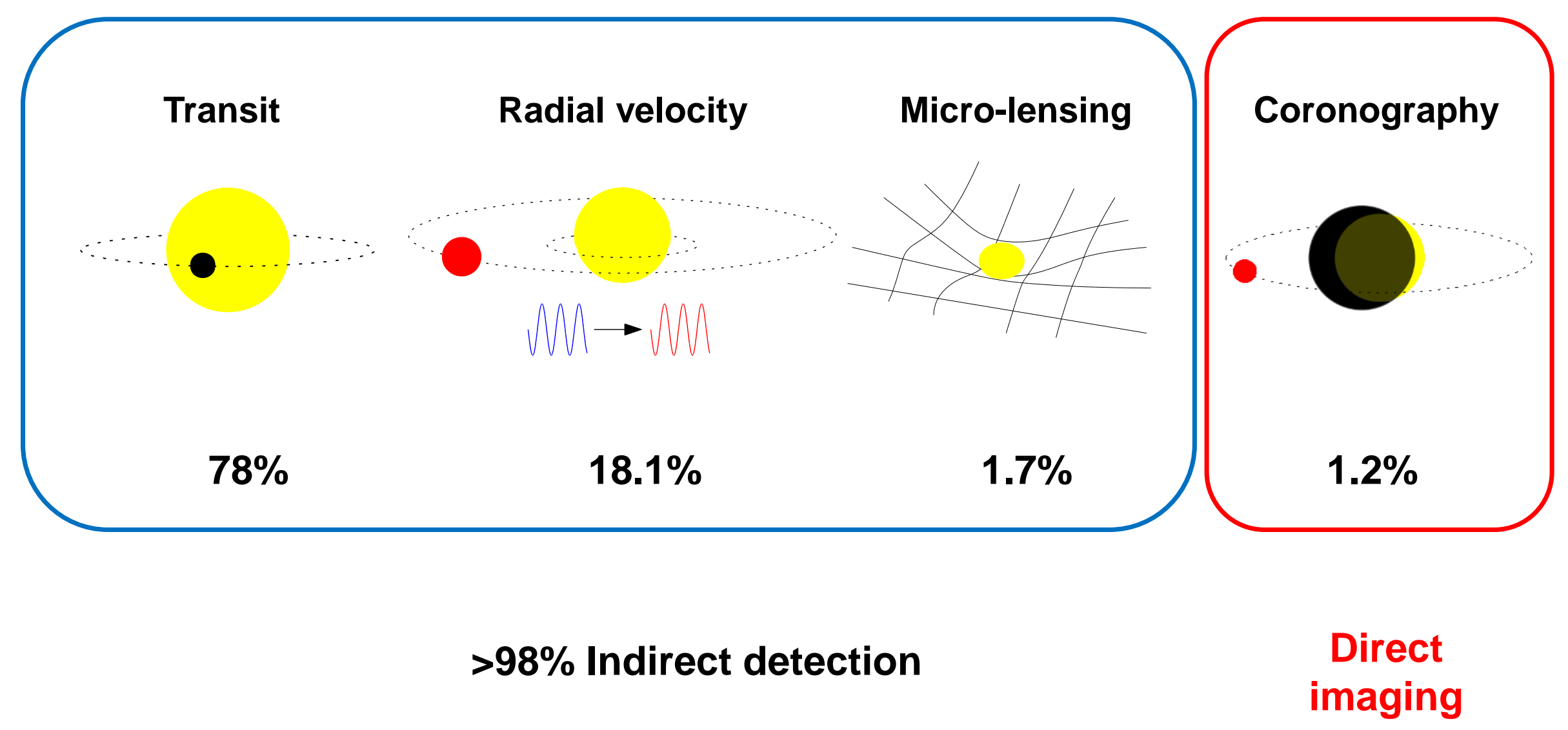




\section{Mass - Period Distribution}

23 Aug 2018

exoplanetarchive.ipoc.caltech.edu

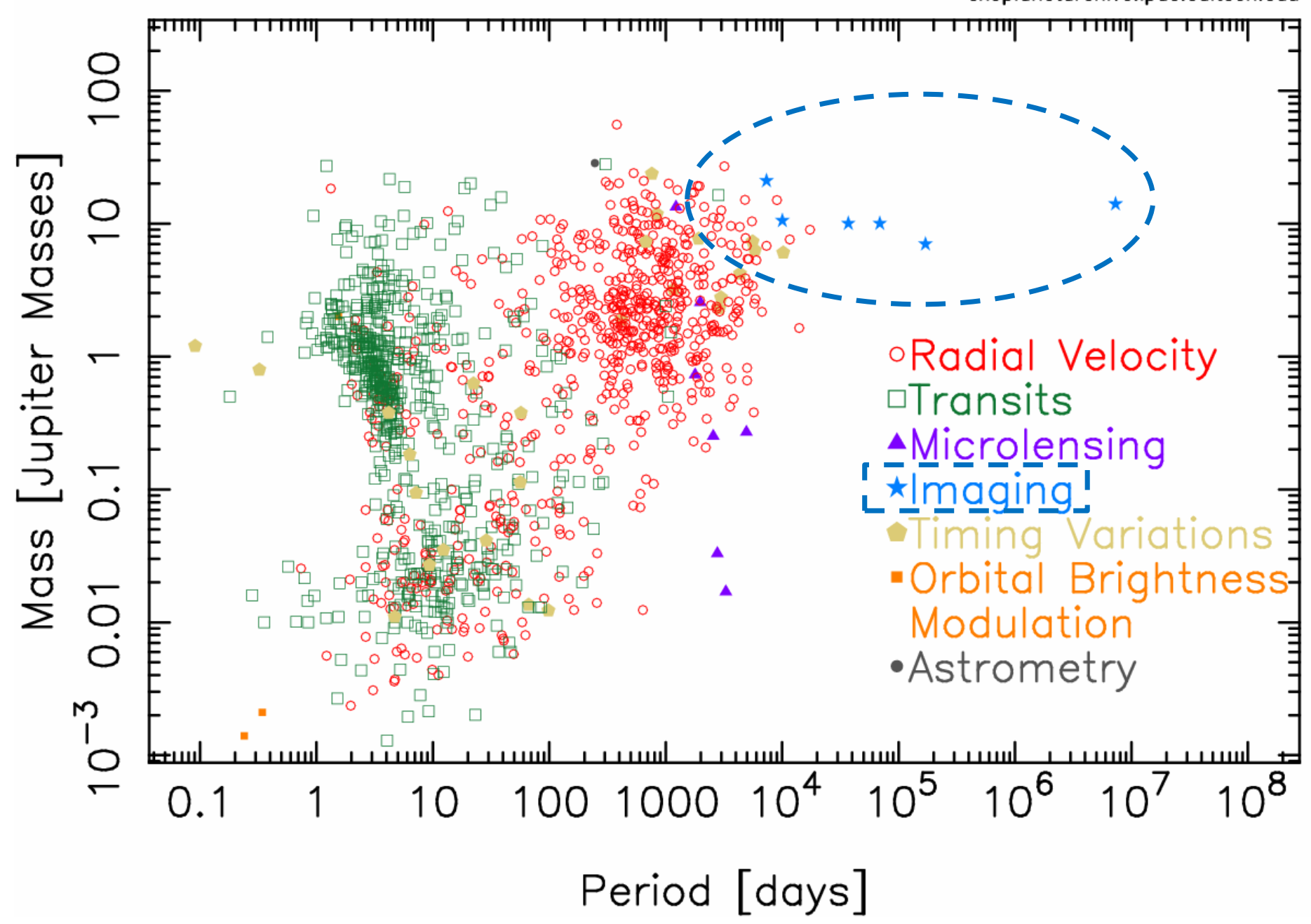




\section{Challenges for direct imaging}

- Contrast: need advanced wavefront and/or phase control techniques

- Angular resolution: need large telescopes (or baselines)

- Sensitivity: need large collecting area

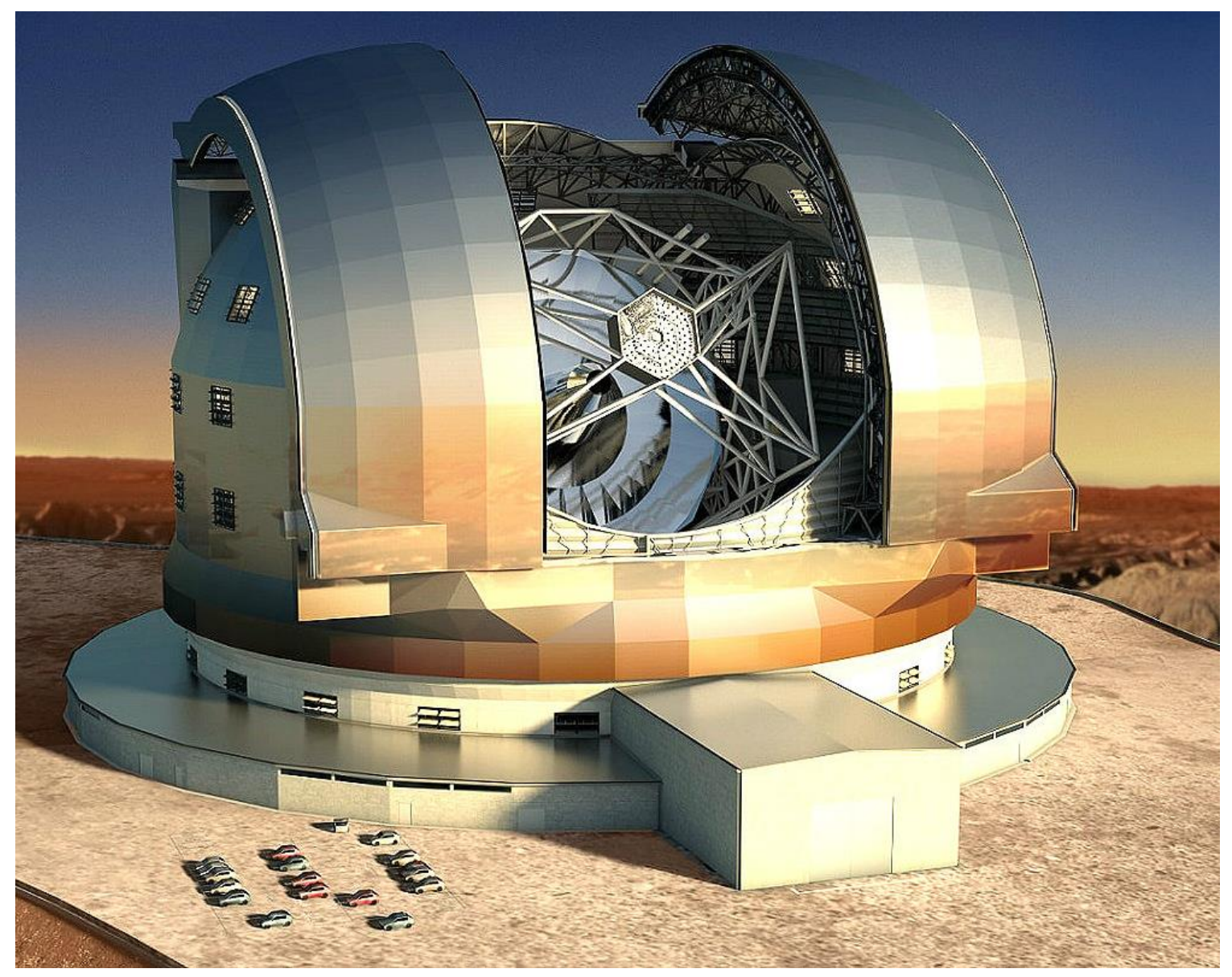



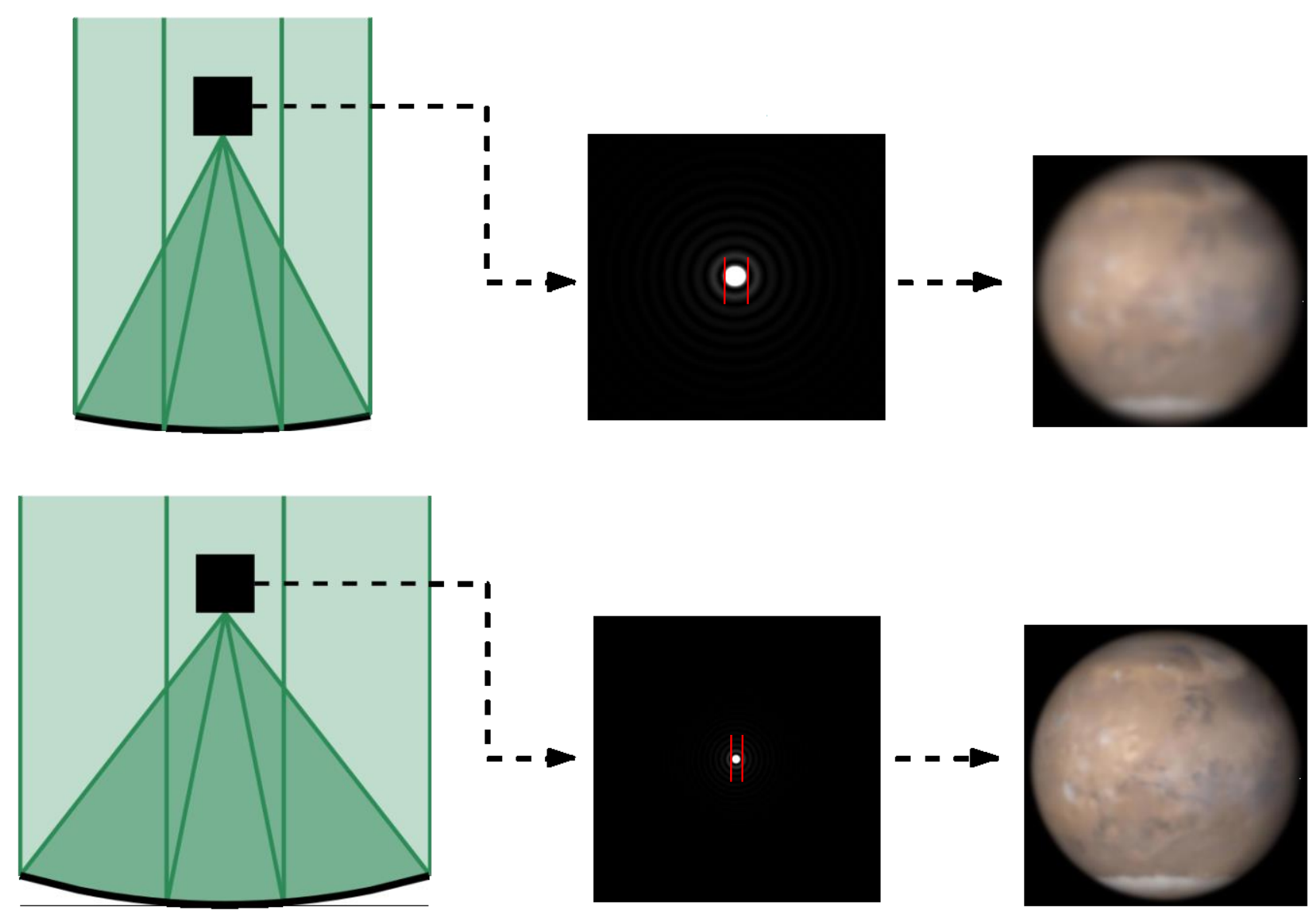


\section{Challenges of exoplanets direct imaging}

How to do direct observation with high contrast and high resolution?

Earth/Sun flux ratio $=10^{-9}$ in the visible
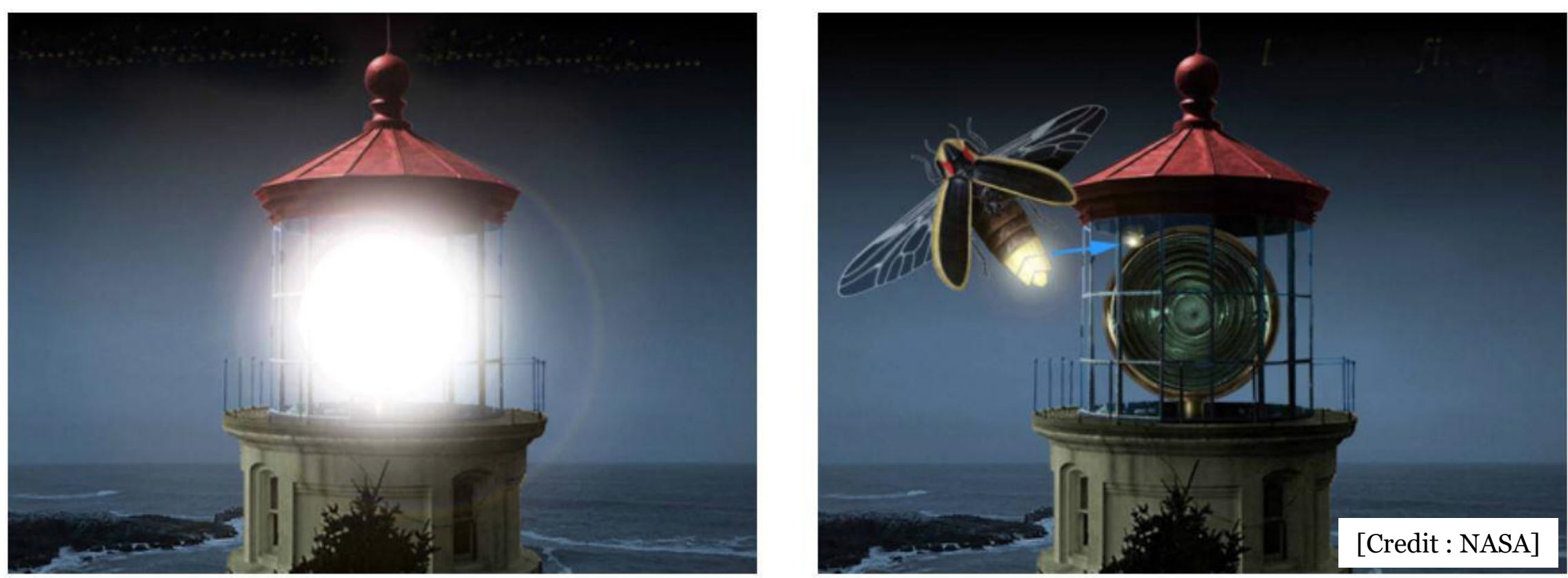

Observation of a firefly close to a lighthouse à $1000 \mathrm{~km}$ 


\section{Interferometry}

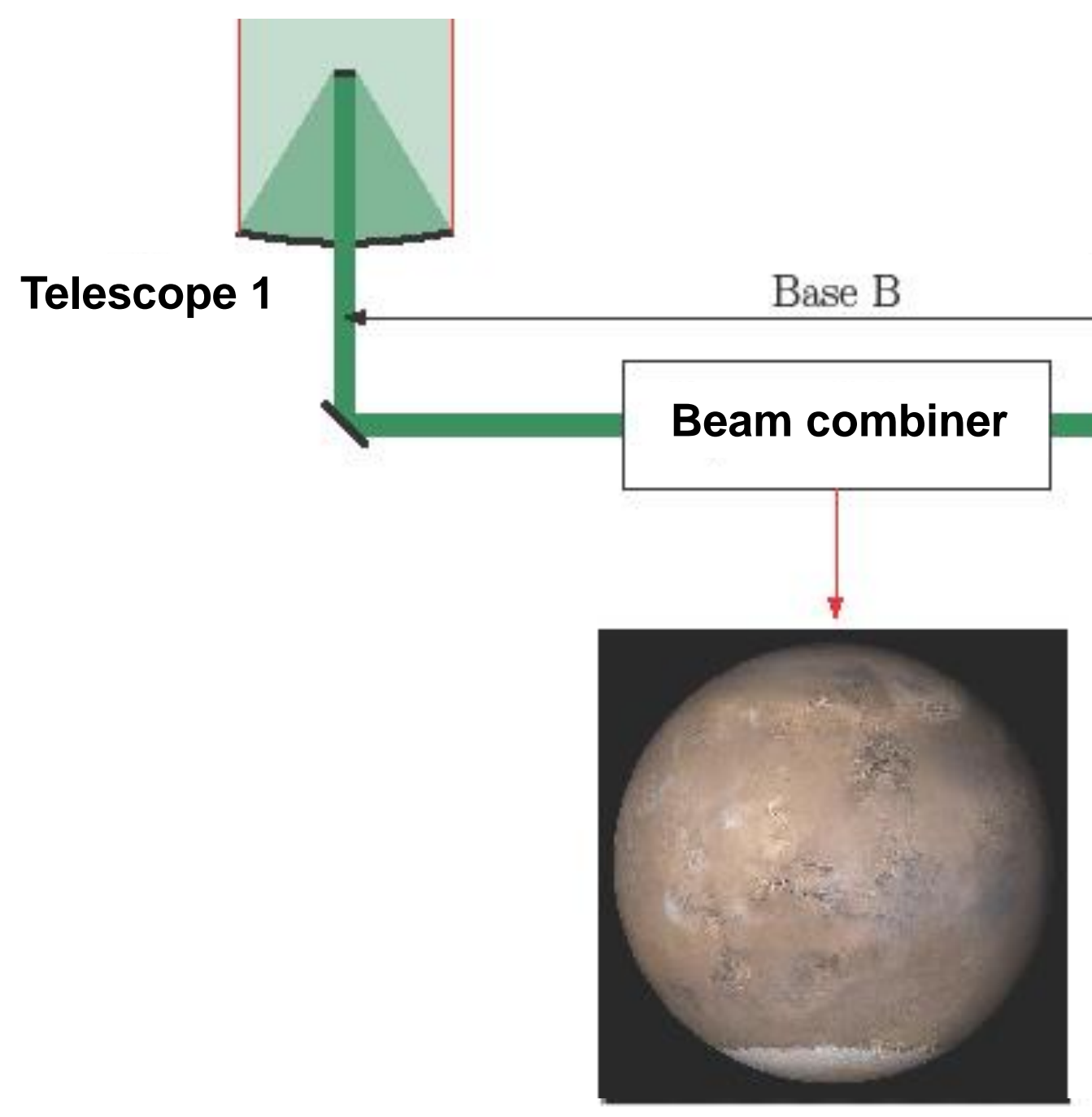

Telescope 2 


\section{Nulling interferometry}

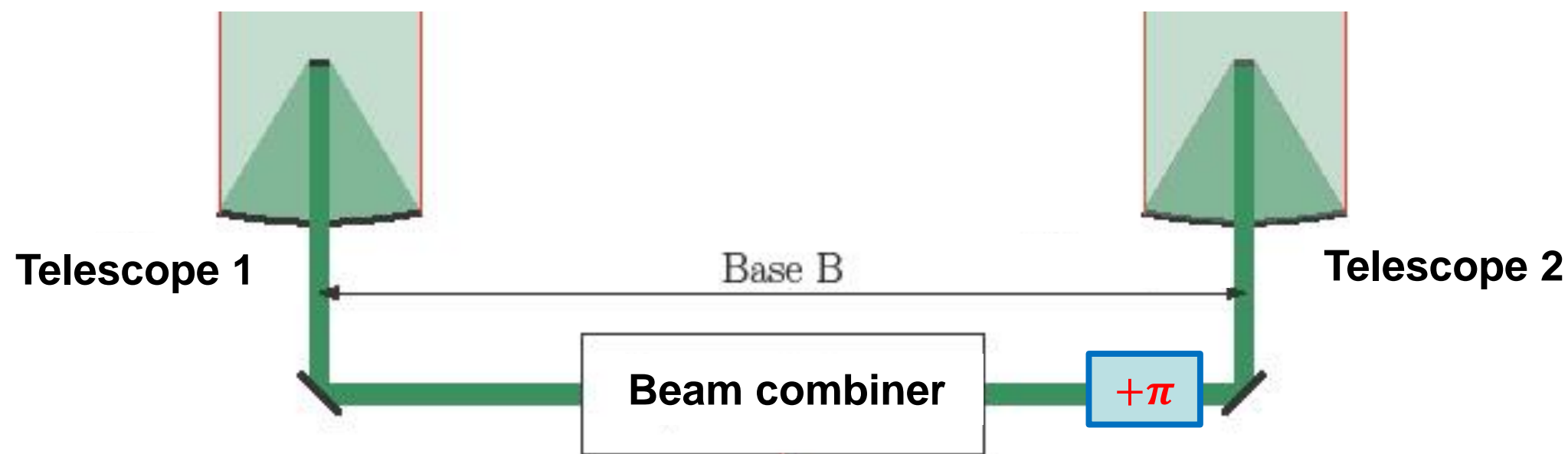

- Bracewell interferometer (1978)

- Introduction of a $\pi$ phase shift to switch-off the star

- Only collect photons from the neighborhood of the $\operatorname{star}(\lambda / 2 B)$

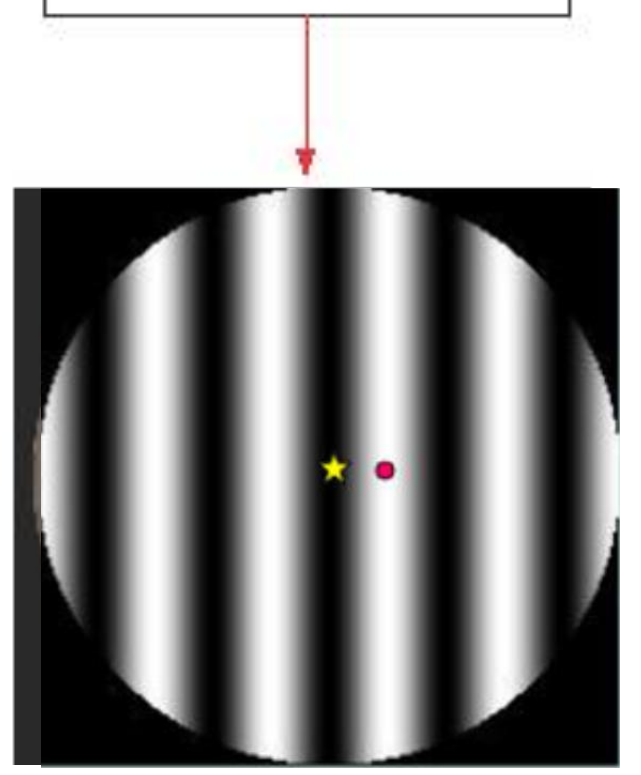




\section{Nulling interferometry}

- Key advantages:

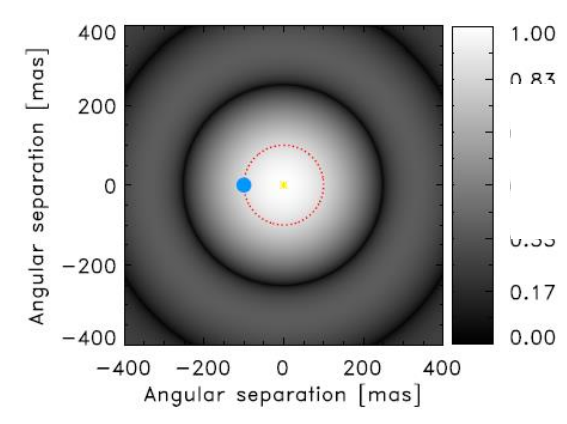

- Interferometry provides the required angular resolution

- Nulling provides the required contrast $\left(\sim 10^{-4}\right.$ already demonstrated from the ground, Menensson et al. 2011, Defrère et al. 2016)
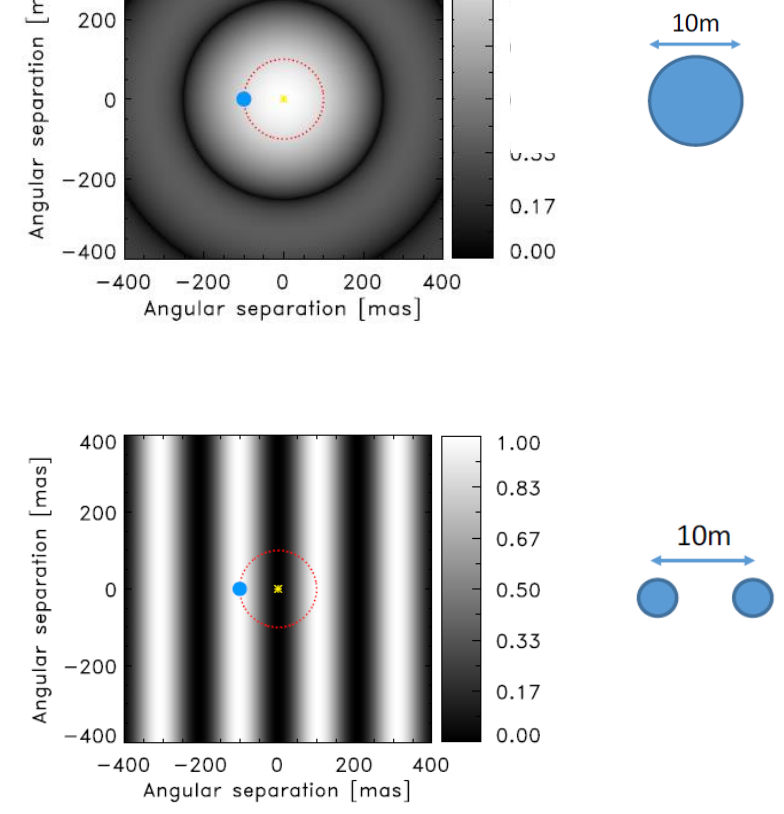

- Must be space-based to get reasonable integration times

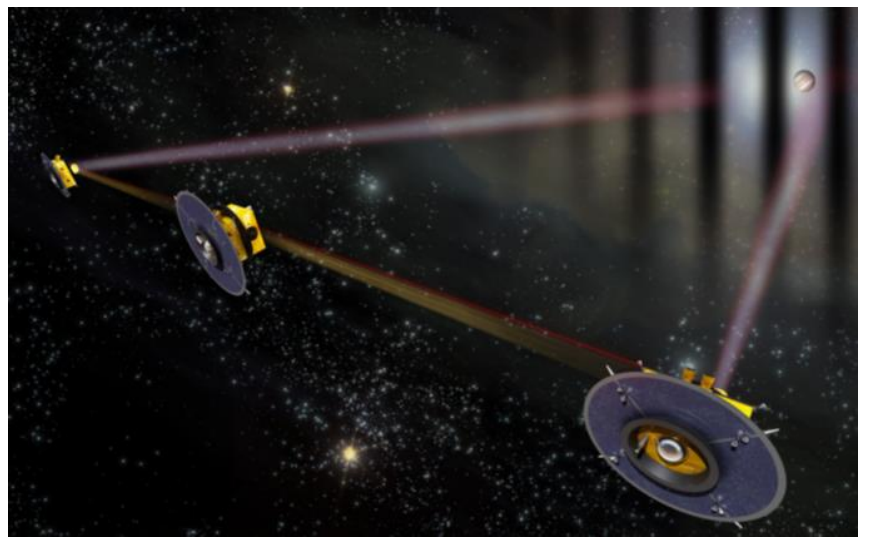




\section{Science Target}

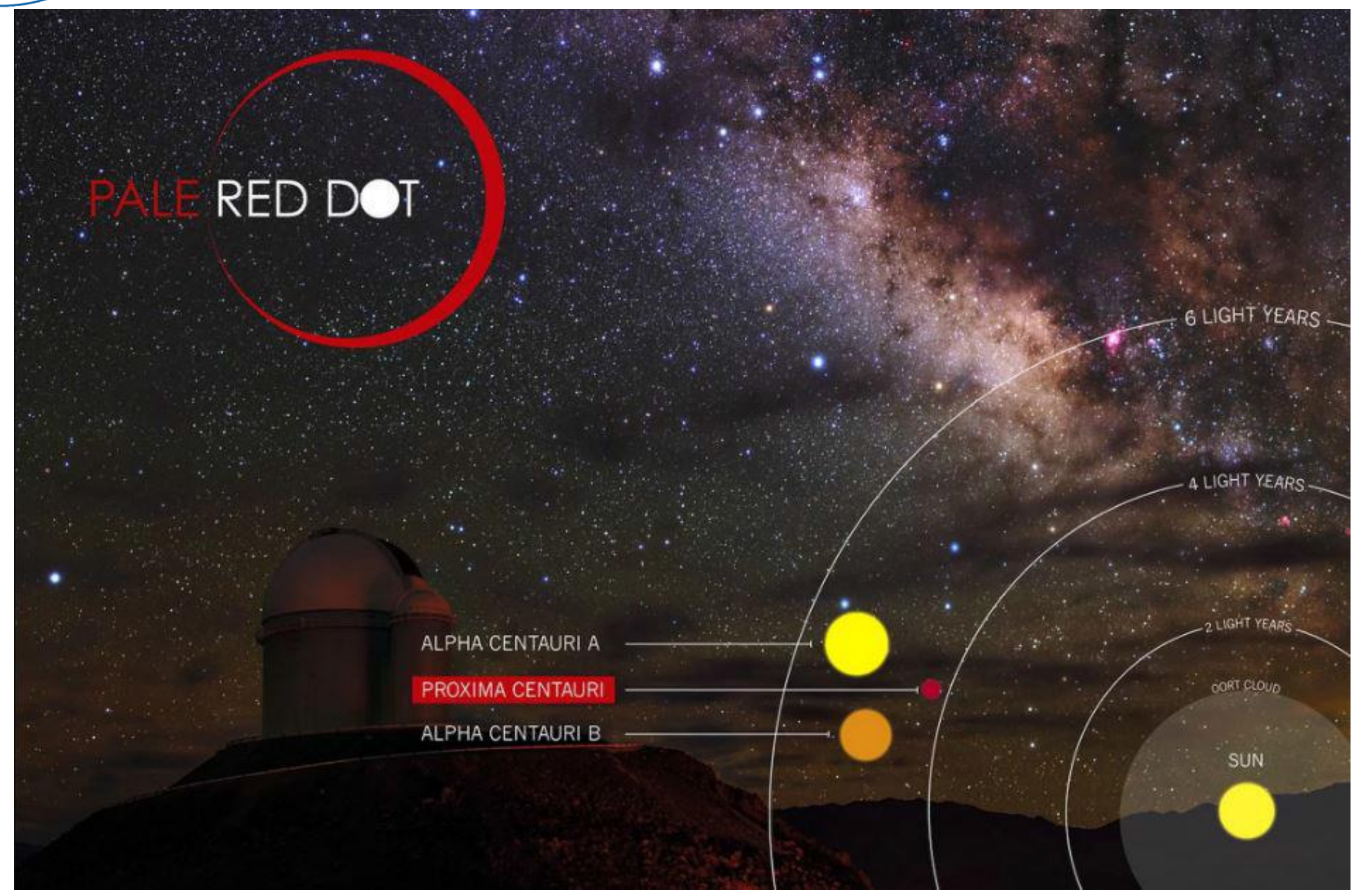




\section{Proxima B}

- Proxima Centauri:

M6V, 1.3 pc (4.2 ly, 270000 UA)

- Detection

a Doppler signal at 11.2 days (Anglada-Escudé et al. Nature 536, 437-440, 2016).
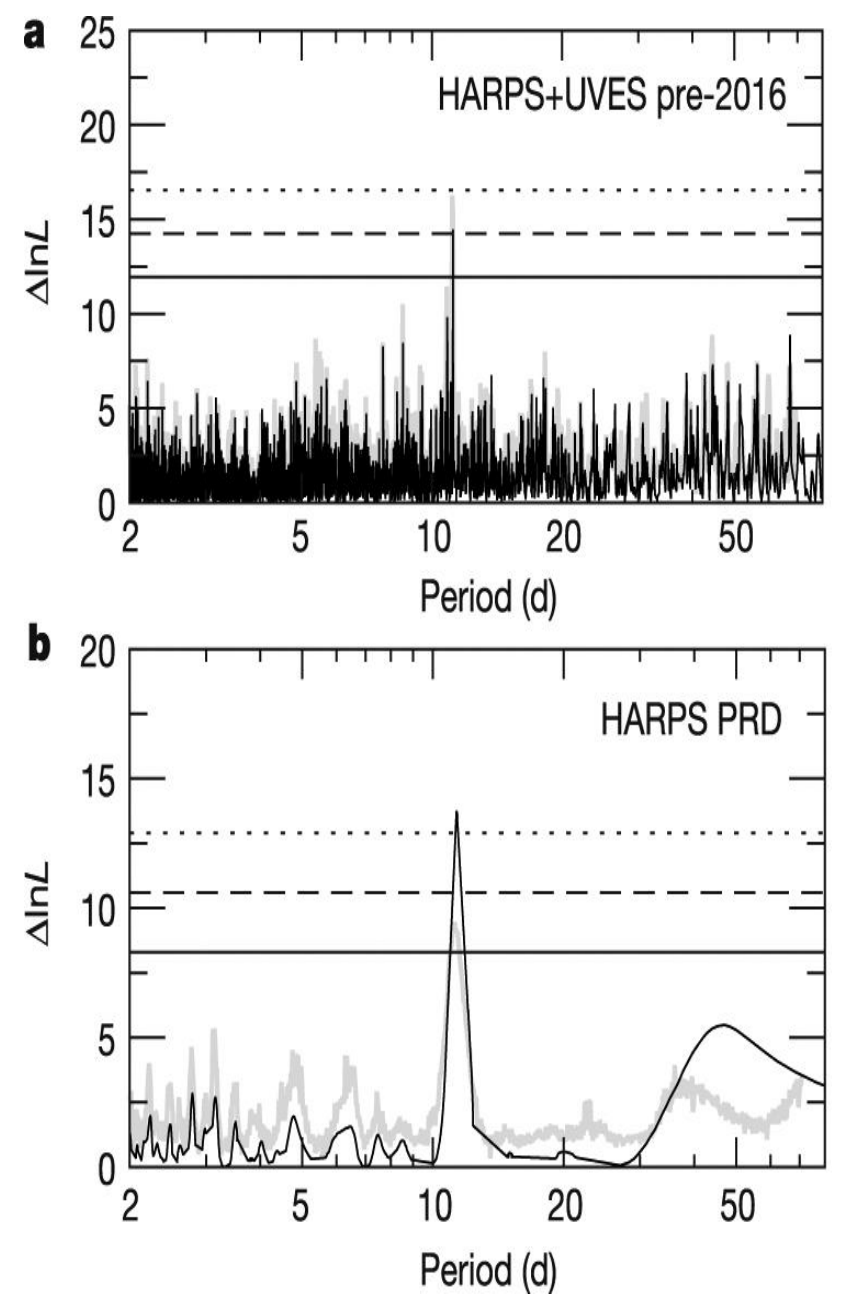

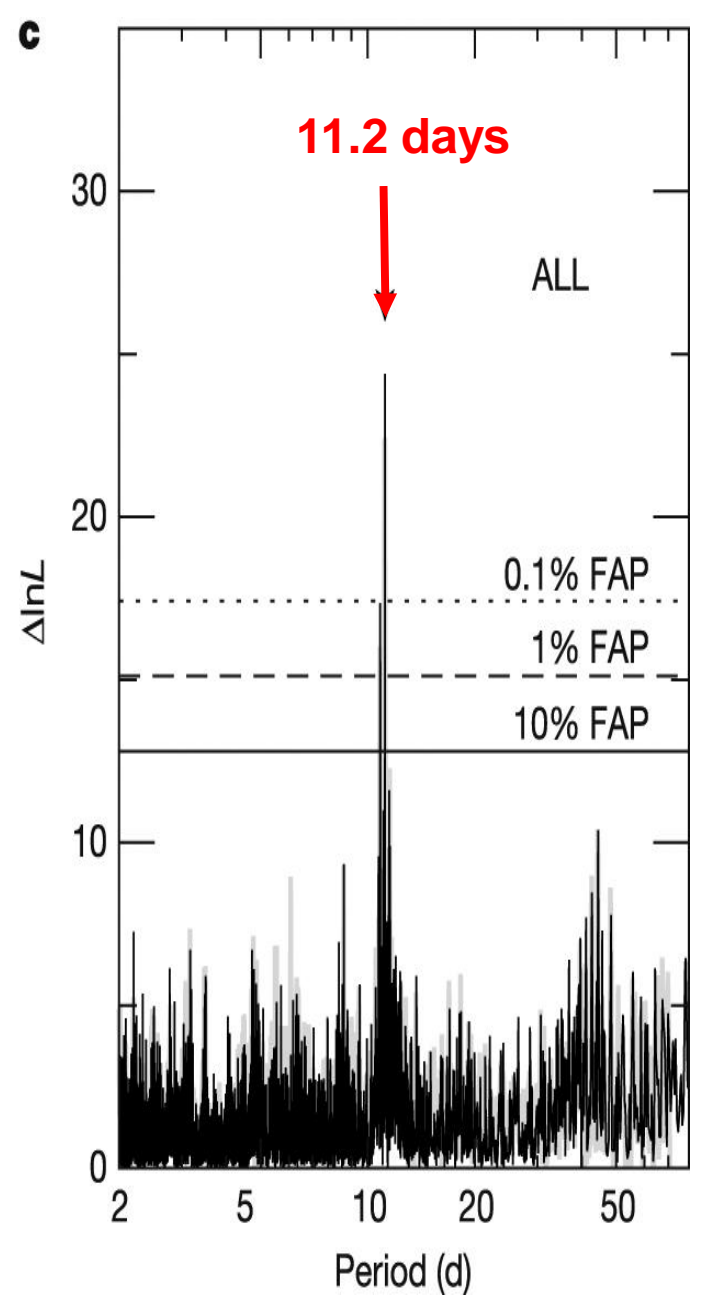




\section{Proxima system}

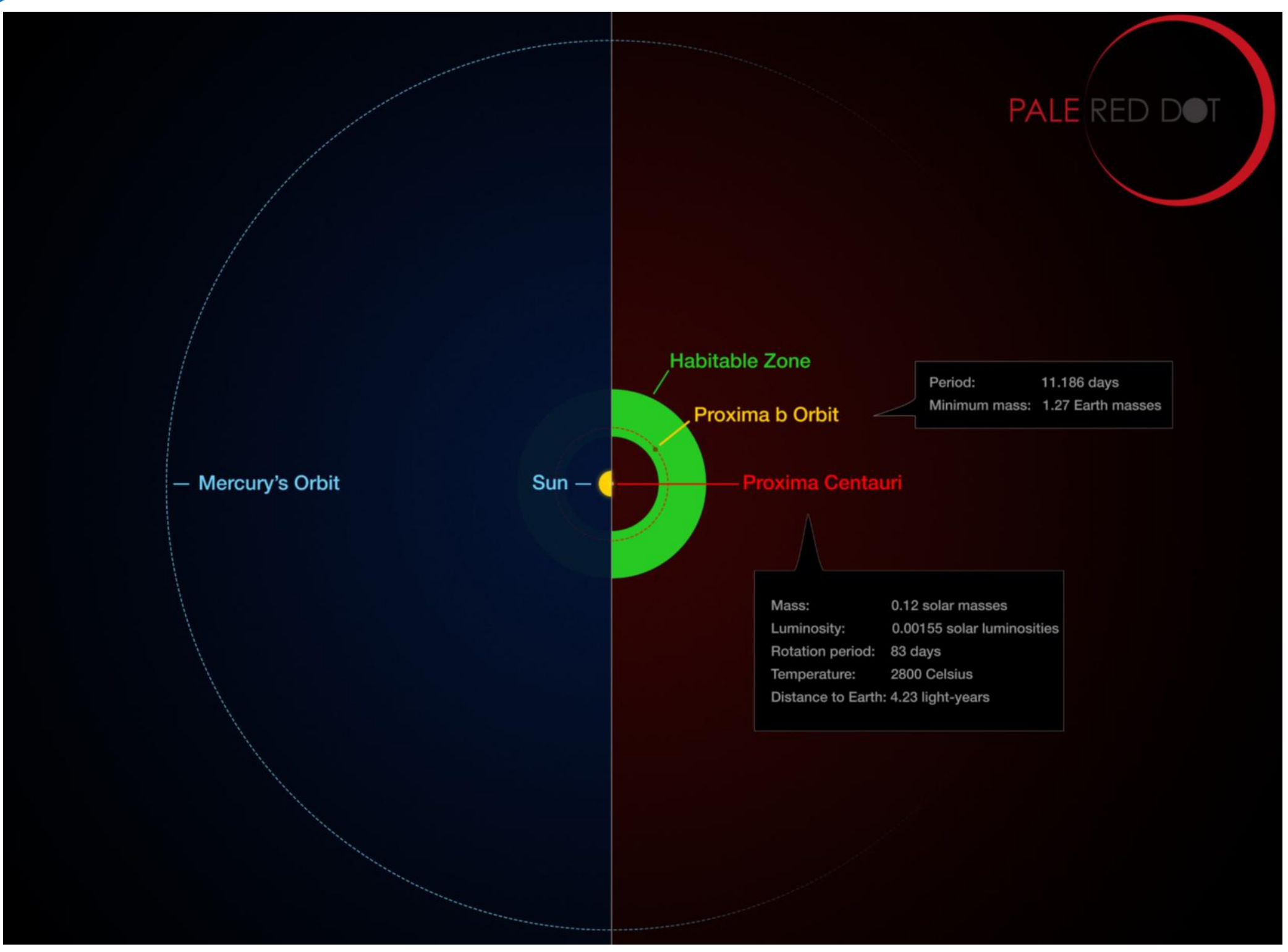




\section{What would be the capabilities of a small platform?}

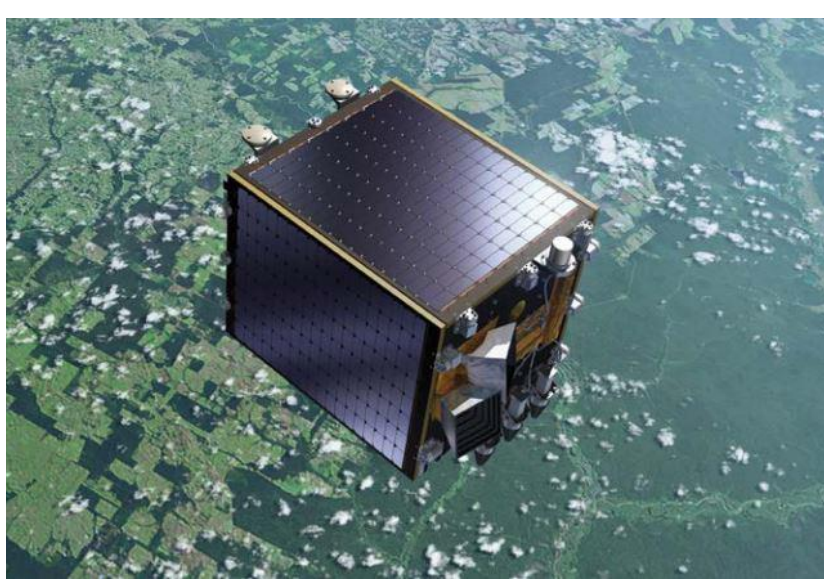

- Methodology

- Apply the Kepler statistics

- Monte-Carlo simulations of a random exoplanets population around Proxima Cen and Alpha Cen

- Definition of the radiated flux from the planet to the Earth

- Define a throughput model of the instrument
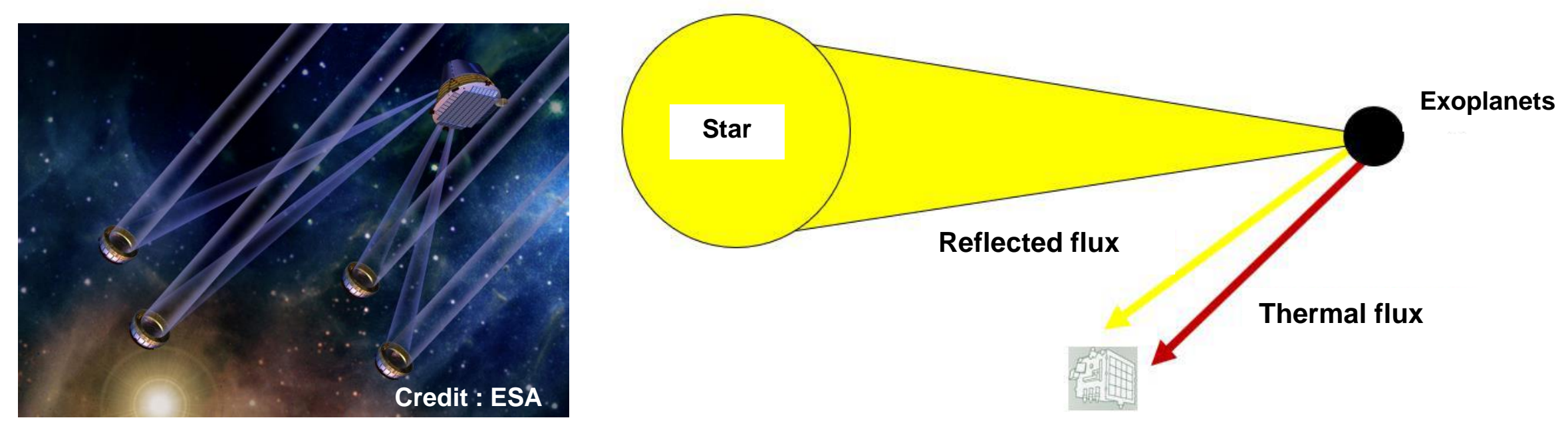

Platform 


\section{What would be the capabilities of a small platform?}

\begin{tabular}{|c|c|c|c|c|c|c|c|c|c|c|c|}
\hline \multirow[t]{2}{*}{ Class } & \multicolumn{11}{|c|}{ Period Range (days) } \\
\hline & $\begin{array}{c}0.8- \\
2.0\end{array}$ & $\begin{array}{c}2.0- \\
3.4\end{array}$ & $\begin{array}{c}3.4- \\
5.9\end{array}$ & $\begin{array}{c}5.9- \\
10\end{array}$ & $\begin{array}{l}10- \\
17\end{array}$ & $\begin{array}{c}17- \\
29\end{array}$ & $\begin{array}{c}29- \\
50\end{array}$ & $\begin{array}{c}50- \\
85\end{array}$ & $\begin{array}{l}85- \\
145\end{array}$ & $\begin{array}{l}145- \\
245\end{array}$ & $\begin{array}{l}245- \\
418^{\mathrm{a}}\end{array}$ \\
\hline \multirow[t]{2}{*}{ Giants } & 0.015 & 0.067 & 0.17 & 0.18 & 0.27 & 0.23 & 0.35 & 0.71 & 1.25 & 0.94 & 1.05 \\
\hline & \pm 0.007 & \pm 0.018 & \pm 0.03 & \pm 0.04 & \pm 0.06 & \pm 0.06 & \pm 0.10 & \pm 0.17 & \pm 0.29 & \pm 0.28 & \pm 0.30 \\
\hline \multirow[t]{2}{*}{ Large Neptunes } & 0.004 & 0.006 & 0.11 & 0.091 & 0.29 & 0.32 & 0.49 & 0.66 & 0.43 & 0.53 & 0.24 \\
\hline & \pm 0.003 & \pm 0.006 & \pm 0.03 & \pm 0.030 & \pm 0.07 & \pm 0.08 & \pm 0.12 & \pm 0.16 & \pm 0.17 & \pm 0.21 & \pm 0.15 \\
\hline \multirow[t]{2}{*}{ Small Neptunes } & 0.035 & 0.18 & 0.73 & 1.93 & 3.67 & 5.29 & 6.45 & 5.25 & 4.31 & 3.09 & $\ldots$ \\
\hline & \pm 0.011 & \pm 0.03 & \pm 0.09 & \pm 0.19 & \pm 0.39 & \pm 0.64 & \pm 1.01 & \pm 1.05 & \pm 1.03 & \pm 0.90 & \\
\hline \multirow[t]{2}{*}{ Super-Earths } & 0.17 & 0.74 & 1.49 & 2.90 & 4.30 & 4.49 & 5.29 & 3.66 & 6.54 & $\ldots$ & $\cdots$ \\
\hline & \pm 0.03 & \pm 0.13 & \pm 0.23 & \pm 0.56 & \pm 0.73 & \pm 1.00 & \pm 1.48 & \pm 1.21 & \pm 2.20 & $\cdots$ & $\cdots$ \\
\hline \multirow[t]{2}{*}{ Earths } & 0.18 & 0.61 & 1.72 & 2.70 & 2.70 & 2.93 & 4.08 & 3.46 & $\ldots$ & $\cdots$ & .. \\
\hline & \pm 0.04 & \pm 0.15 & \pm 0.43 & \pm 0.60 & \pm 0.83 & \pm 1.05 & \pm 1.88 & \pm 2.81 & & & \\
\hline \multirow[t]{2}{*}{ Total } & 0.41 & 1.60 & 4.22 & 7.79 & 11.2 & 13.3 & 16.7 & 13.7 & & F & al" \\
\hline & \pm 0.05 & \pm 0.20 & \pm 0.50 & \pm 0.85 & \pm 1.2 & \pm 1.6 & \pm 2.6 & \pm 3.2 & & & \\
\hline
\end{tabular}

Radius - Period Distribution

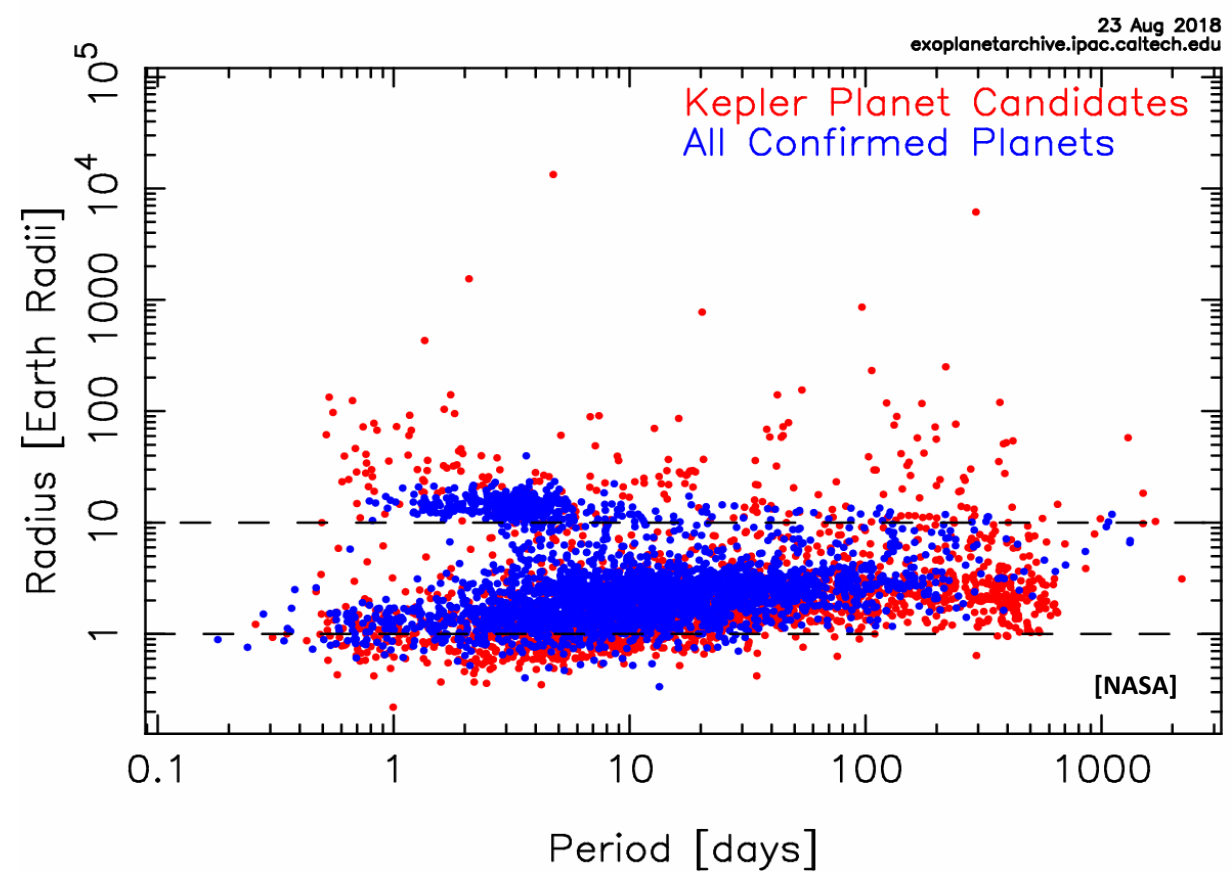




\section{Simulated of exoplanets around Alpha Cen}

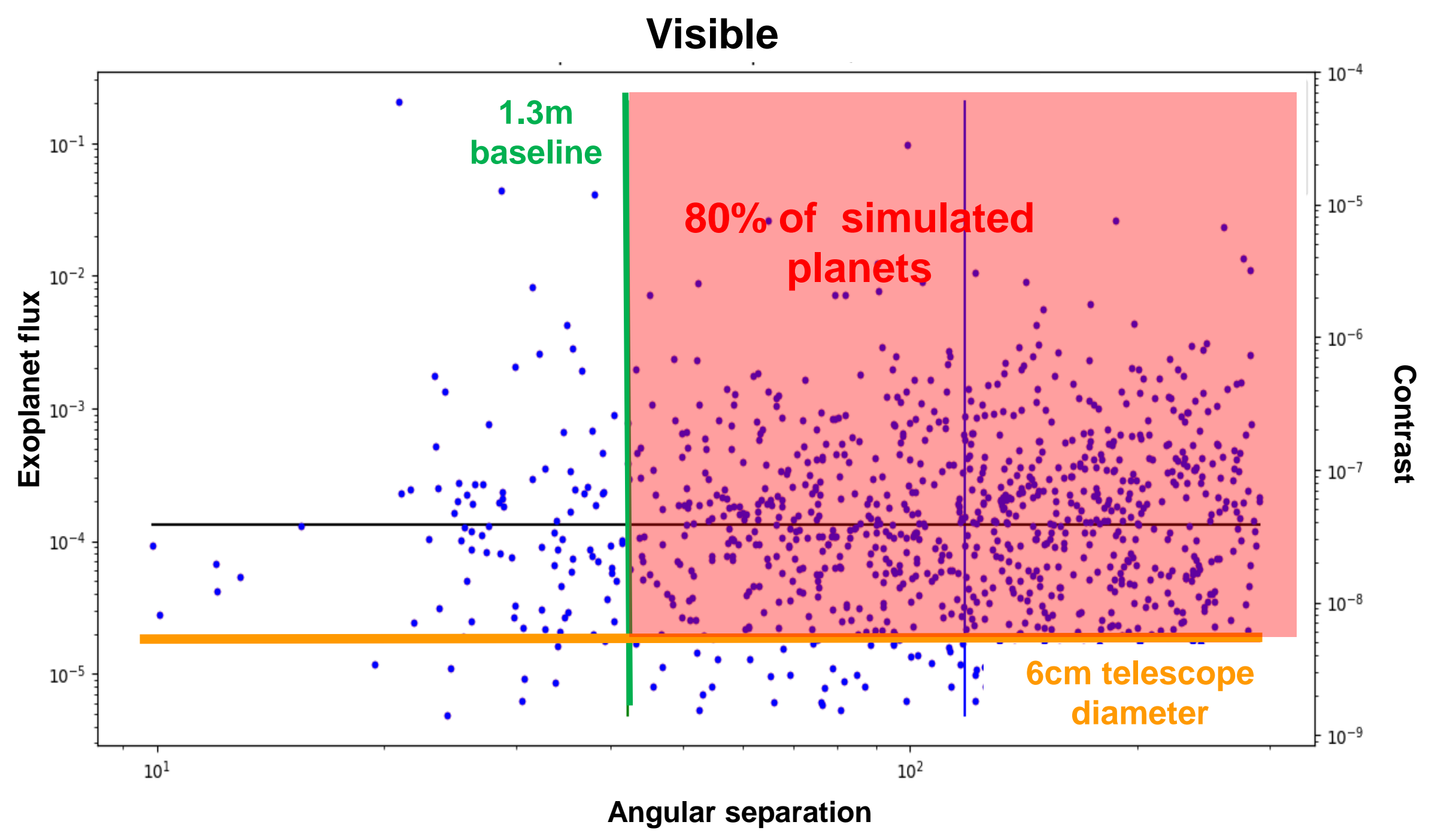




\section{Simulated of exoplanets around Proxima Cen}

\section{Visible}

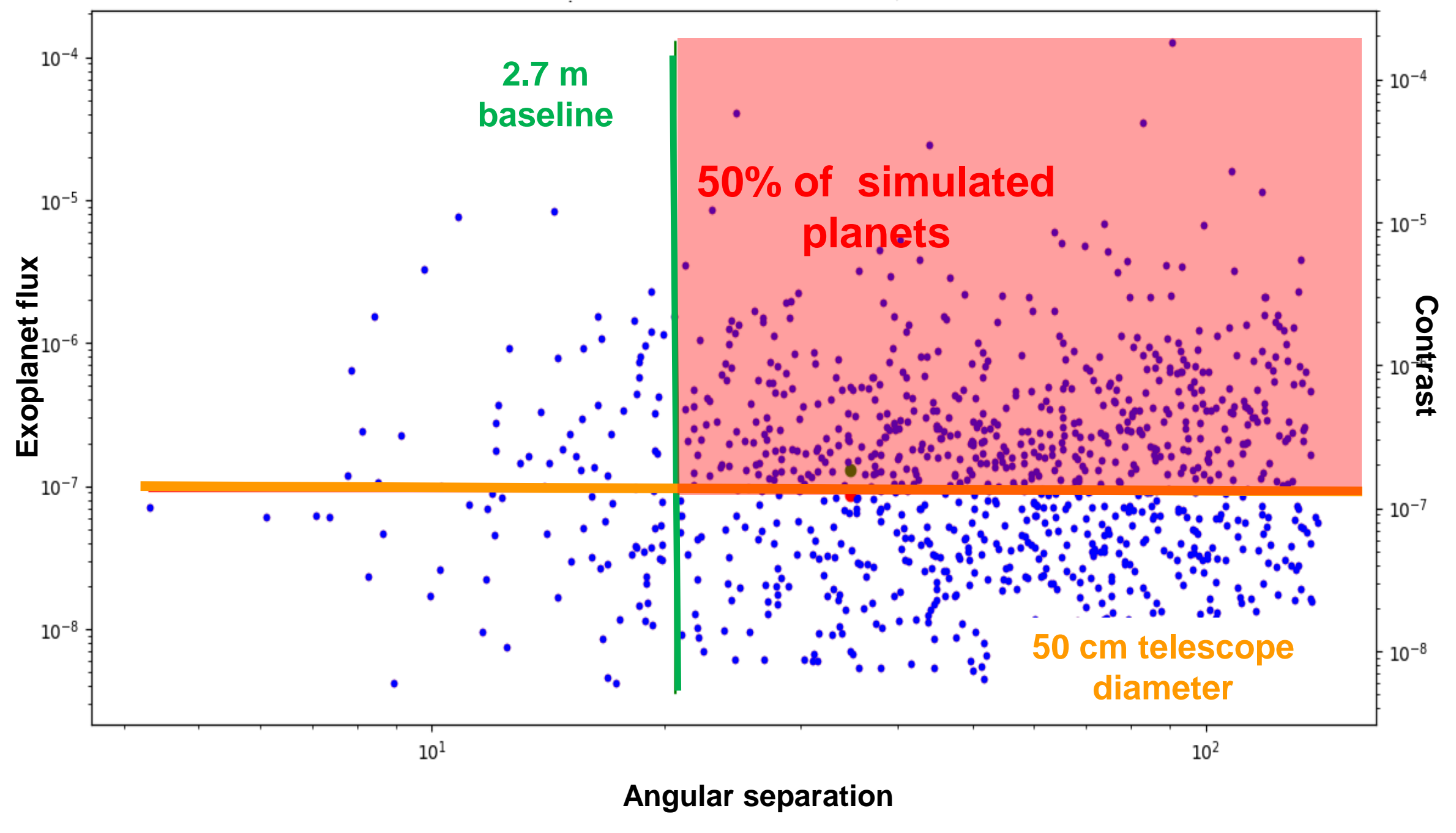




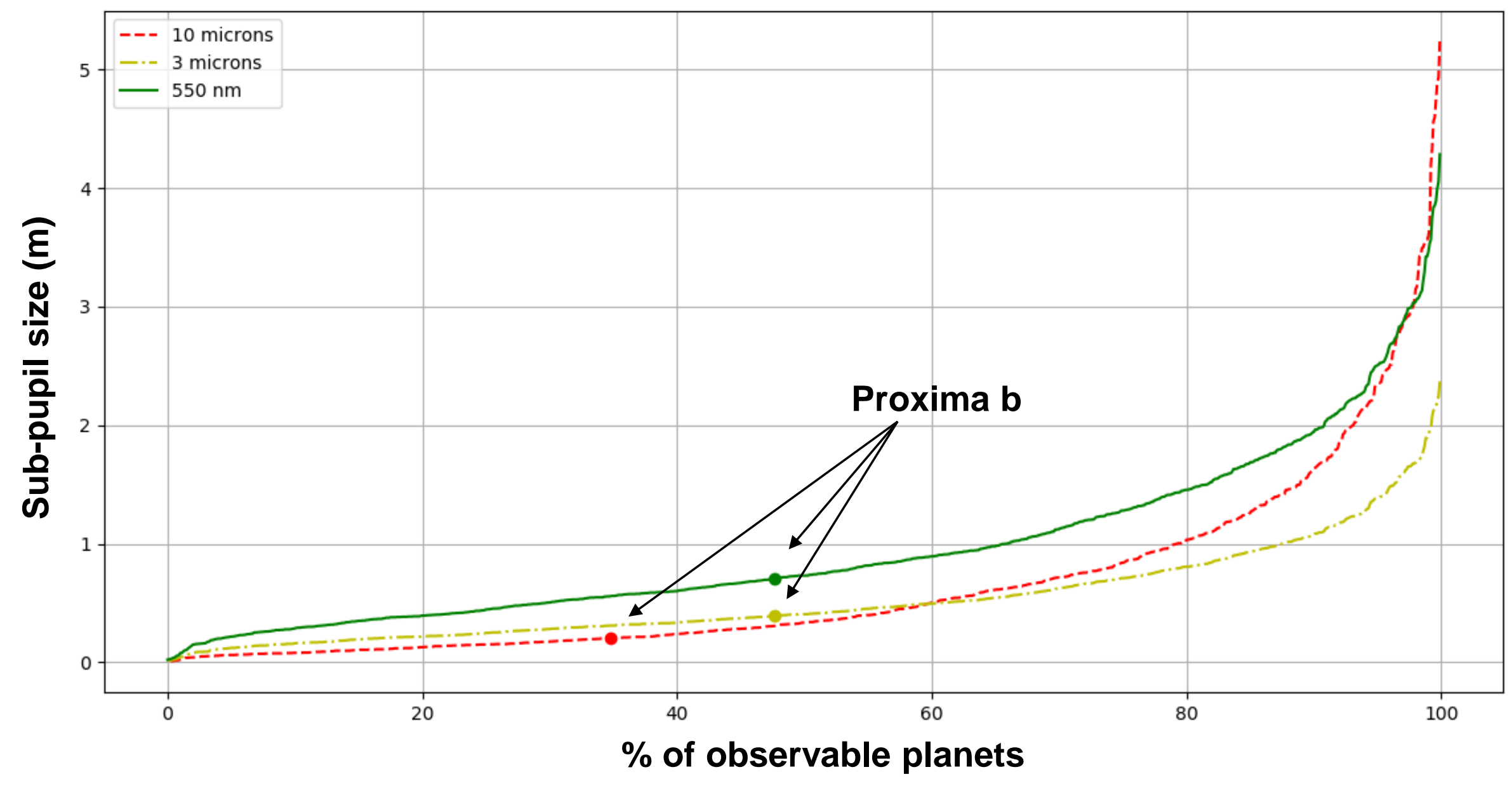




\begin{tabular}{|c|c|c|c|c|}
\hline & \multicolumn{2}{|c|}{ Alpha Cen } & \multicolumn{2}{c|}{ Proxima Cen } \\
\hline & $\begin{array}{c}\text { Base } \\
(\mathrm{m})\end{array}$ & $\begin{array}{c}\text { Telescope } \\
\text { Diameter } \\
(\mathbf{m})\end{array}$ & $\begin{array}{c}\text { Base } \\
(\mathrm{m})\end{array}$ & $\begin{array}{c}\text { Telescope } \\
\text { Diameter } \\
(\mathbf{m})\end{array}$ \\
\hline Visible & 1.3 & 0.06 & 2.7 & 2 \\
\hline $\mathbf{3} \mathbf{\mu m}$ & 7 & 0.09 & 15 & 1.1 \\
\hline $\mathbf{1 0} \mathbf{\mu m}$ & 25 & 0.06 & 50 & 1.3 \\
\hline
\end{tabular}
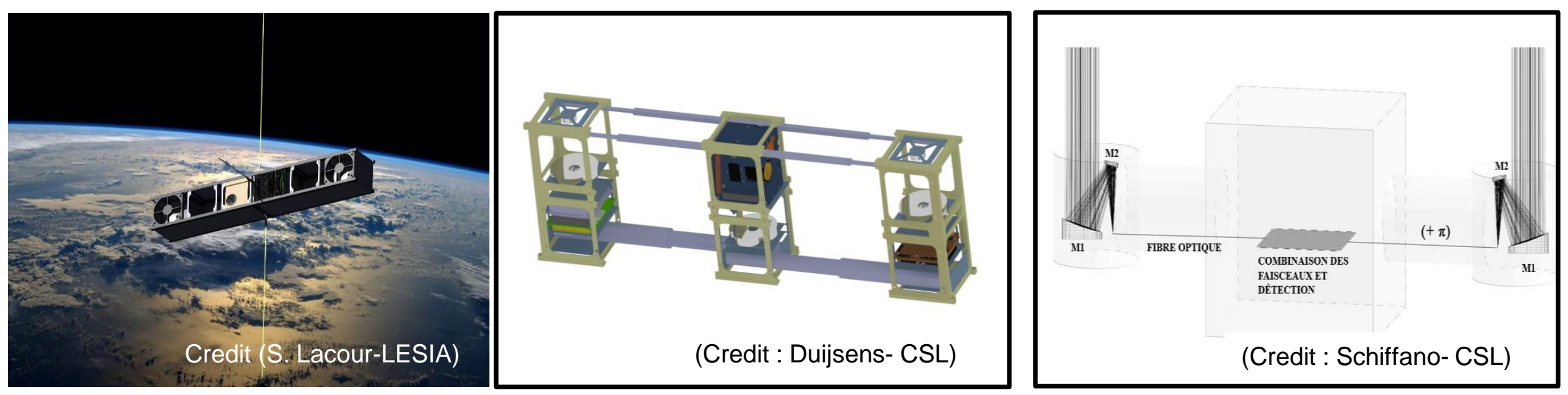


\section{Take home messages}
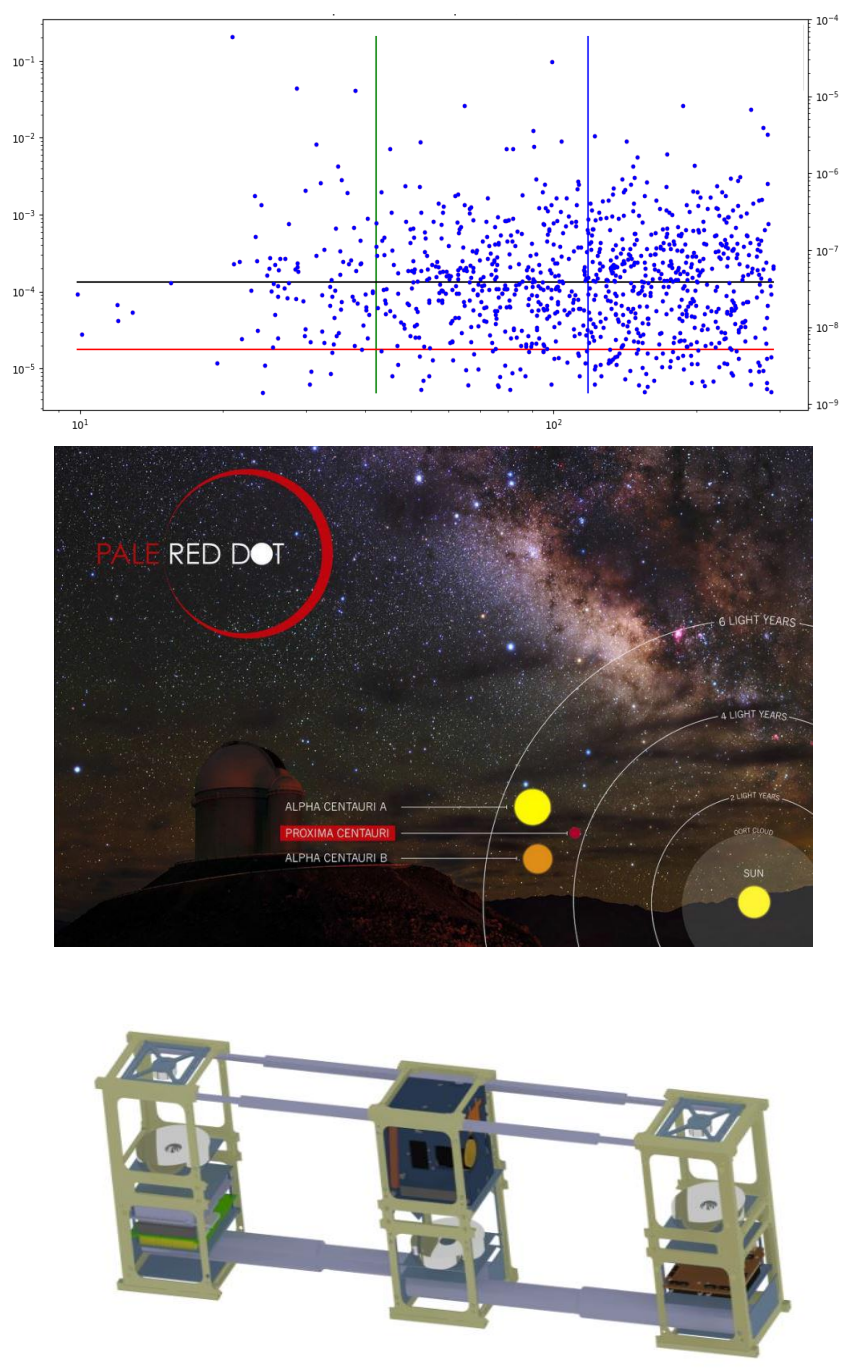

- The interferometry is a very powerful technique well proven on ground

- solution to improve drastically the angular resolution

- Improve the contrast sensitivity

- Mission concept is

- Science case focused on Alpha centauri system with the goal of observing exoplanets

- Characterise Proxima B

- Technology demonstrator to be a pathfinder to larger interferometric mission for space 


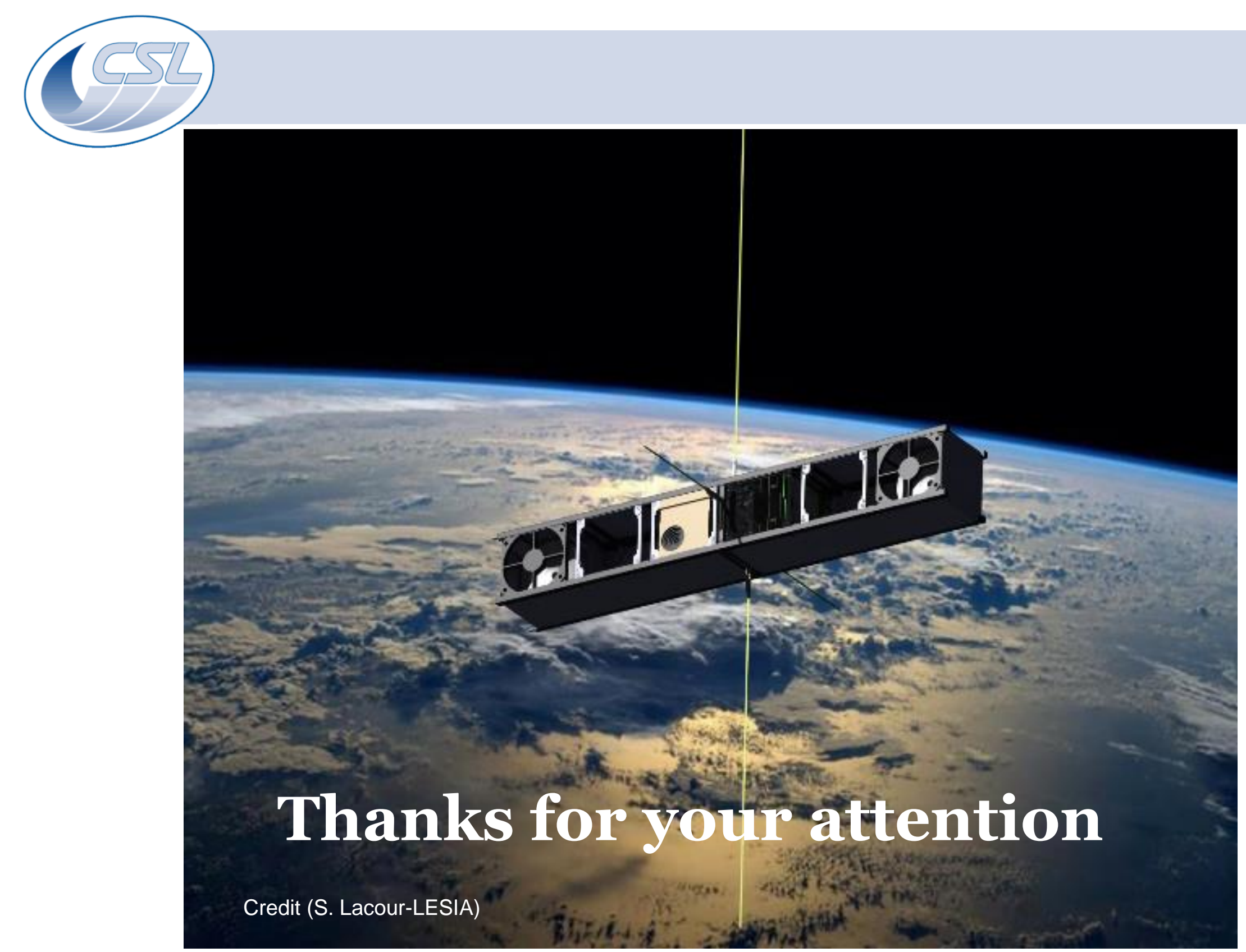

